\title{
The Generalized Odd Generalized Exponential Family of Distributions: Properties, Characterizations and Application
}

\author{
Morad Alizadeh ${ }^{1}$, Indranil Ghosh ${ }^{2}$, Haitham M. Yousof ${ }^{3}$, \\ Mahdi Rasekhi ${ }^{4}$, G.G.Hamedani ${ }^{5}$ \\ ${ }^{I}$ Department of Statistics, Faculty of Sciences, Persian Gulf University, Iran \\ ${ }^{2}$ Department of Mathematics and Statistics, University of North Carolina, USA \\ ${ }^{3}$ Department of Statistics, Mathematics and Insurance, Benha University, Egypt \\ ${ }^{4}$ Department of Statistics, Malayer University, Iran \\ ${ }^{5}$ DepartmentofMathematics, Statistics and Computer Science, Marquette University, USA
}

\begin{abstract}
We introduce a new class of distributions called the generalized odd generalized exponential family. Some of its mathematical properties including explicit expressions for the ordinary and incomplete moments, quantile and generating functions, Rényi, Shannon and q-entropies, order statistics and probability weighted moments are derived. We also propose bivariate generalizations. We constructed a simple type Copula and intro-duced a useful stochastic property. The maximum likelihood method is used for estimating the model parameters. The importance and flexibility of the new family are illustrated by means of two applications to real data sets. We assess the performance of the maximum likelihood estimators in terms of biases and mean squared errors via a simulation study.
\end{abstract}

Key words: Characterizations, Generalized Odd Generalized exponential-G Family, Generating Function, Maximum Likelihood Estimation, Moments, Quantile function.

\section{Introduction}

The statistical literature contains many new classes of distributions that have been constructed by extending the common families of continuous distributions providing more flexibility as far as applications is concerned. These new families have been used for modeling data in many applied areas such as engineering, economics, biological studies and environmental sciences. However, applied areas such as lifetime analysis, finance and insurance clearly require extended forms of these distributions. So, several classes of distributions have been constructed by extending common families of continuous distributions. These generalized distributions give more flexibility by adding new parameters to the baseline model. These methods were pioneered by Gupta et al. (1998) who proposed the exponentiated-G class, which consists of raising the cumulative distribution function (cdf) to a positive power parameter. Many other classes can be cited such as the Marshall-Olkin-G family by Marshall and Olkin (1997), beta generalized-G family by Eugene et al. (2002), exponentiated generalized-G family by Cordeiro et al. (2013), a new method for generating families of continuous distributions by Alzaatreh et al. (2013), transmuted exponentiated generalized-G by Yousof et al. 
(2015), exponentiated transmuted-G by Merovc et al. (2016), Burr X-G by Yousof et al. (2016), Odd-Burr generalized family by Alizadeh et al (2016a), transmuted Weibull G family by Alizadeh et al. (2016b), complementary generalized transmuted Poisson-G family by Alizadeh et al. (2016b), Kumaraswamy transmuted-G by Afify et al. (2016c), complementary geometric transmuted-G family Afify et al. (2016b), transmuted geometric-G by Afify et al. (2016a), generalized transmuted-G by Nofal et al. (2017), exponentiated generalized-G Poisson by Aryal and Yousof (2017), Marshall-Olkin generalized family by Yousof et al. (2017a), beta Weibull-G family of distributions by Yousof et al. (2017b), Topp-Leone odd log-logistic family by de Brito et al. (2017), Type I general exponential class of distributions by Hamedani et al. (2017), exponentiated Weibull-H family Cordeiro et al. (2017a), Burr XII system of densities by Cordeiro et al. (2017b) and beta transmuted-H family by Afify et al. (2017), among others. In this paper, we propose and study a new generated family called the Generalized Odd Generalized Exponential-G (GOGE-G) family via the T-X family defined by Alzaatreh et al. (2013) and give a comprehensive description of its mathematical properties. In fact, the GOGE-G family is motivated by its flexibility in application. By means of two applications, it is shown that the GOGE-G class provides better fits than at least ten other families each having the same num-ber of parameters. The cumulative distribution function (cdf) and probability desity function (pdf) of GOGE-G class are given, respectively by

$$
\begin{gathered}
F(x)=\left[1-e^{\frac{-G(x ; \psi)^{\alpha}}{1-G(x ; \psi)^{\alpha}}}\right]^{\beta}, \\
f(x)=\frac{\alpha \beta g(x ; \psi) G(x ; \psi)^{\alpha-1}}{\left[1-G(x ; \psi)^{\alpha}\right]^{2}} e^{\frac{-G(x ; \psi) \alpha}{1-G(x ; \psi)^{\alpha}}}\left[1-e^{\frac{-G(x ; \psi)^{\alpha}}{1-G(x ; \psi)^{\alpha}}}\right]^{\beta-1},
\end{gathered}
$$

where $\mathrm{G}(\mathrm{x} ; \psi)$ is the baseline cdf depending on a parameter vector $\psi$ and $\mathrm{g}(\mathrm{x} ; \psi)$ is its corresponding pdf and $\alpha, \beta>0$ are two additional shape parameters. Henceforth, $\mathrm{X} \sim \mathrm{GOGE}-\mathrm{G}(\alpha, \beta, \psi)$ denotes a random variable having density function

(2). The reliability function (rf), hazard rate function (hrf), reversed hazard rate (rhr) and cumulative hazard rate function (chrf) of $\mathrm{X}$ are given, respectively, by

$$
R(x)=1-\left[1-e^{\frac{-G(x ; \psi)^{\alpha}}{1-G(x ; \psi)^{\alpha}}}\right]^{\beta}, h(x)=\frac{\alpha \beta g(x ; \psi) G(x ; \psi)^{\alpha-1} e^{\frac{-G(x ; \psi)^{\alpha}}{1-G(x ; \psi)^{\alpha}}}\left[1-e^{\frac{-G(x ; \psi)^{\alpha}}{1-G(x ; \psi)^{\alpha}}}\right]^{\beta-1}}{\left[1-G(x ; \psi)^{\alpha}\right]^{2}\left\{1-\left[1-e^{\frac{-G(x ; \psi)^{\alpha}}{1-G(x ; \psi)^{\alpha}}}\right]^{\beta}\right\}} .
$$

For simulating data from this family, if $\mathrm{u} \sim \mathrm{u}(0,1)$, then

$$
x_{u}=G^{-1}\left\{\left[\frac{-\log \left(1-u^{\frac{1}{\beta}}\right)}{1-\log \left(1-u^{\frac{1}{\beta}}\right)}\right]^{\frac{1}{\alpha}} ; \psi\right\}
$$

has cdf (1). We omit sometimes the dependence on the parameters and simply use, $\mathrm{g}(\mathrm{x})=\mathrm{g}(\mathrm{x} ; \psi)$ and $\mathrm{G}(\mathrm{x})=\mathrm{G}(\mathrm{x} ; \psi)$. Now, we provide a useful representation for (2). Using the series expansion $(1-z)^{\beta-1}=\sum_{i=0}^{\infty} \frac{(-1)^{i} \Gamma(\beta)}{i ! \Gamma(\beta-i)} z^{i}$, which holds for $|\mathrm{z}|<$ 
1 and $b>0$ real non-integer and using the power series, the pdf of the GOGE-G family in (2) can be expressed as

$$
f(x)=\alpha \beta g(x) \sum_{i, j=0}^{\infty} \frac{(-1)^{i+j}(i+1)^{j}\left(\begin{array}{c}
\beta-1 \\
i
\end{array}\right)}{j !\left[1-G(x)^{\alpha}\right]^{j+2}} G(x)^{\alpha(j+1)-1} .
$$

Using the series expansion again we arrive at

$$
f(x)=\sum_{j, k=0}^{\infty} w_{j, k} h_{\alpha(j+k+1)}(x),
$$

where $\mathrm{h}_{\gamma}(\mathrm{x})=\gamma \mathrm{g}(\mathrm{x}) \mathrm{G}(\mathrm{x})^{\gamma-1}$ represents the pdf of the exp-G family with power parameter $\gamma$ and

$$
w_{j, k}=\frac{\alpha \beta(-1)^{j+k}\left(\begin{array}{c}
-(j+2) \\
k
\end{array}\right)}{j ![\alpha(j+k+1)]} \sum_{i=0}^{\infty}(-1)^{i}(i+1)^{j}\left(\begin{array}{c}
\beta-1 \\
i
\end{array}\right) .
$$

The cdf of the GOGE-G family can also be expressed as a mixture of exp-G cdfs. By integrating (4), we obtain the same mixture representation

$$
F(x)=\sum_{j, k=0}^{\infty} w_{j, k} H_{\alpha(j+k+1)}(x),
$$

where $\mathrm{H} \gamma(\mathrm{x})$ is the cdf of the exp-G family with power parameter $\gamma$.

The chief motivation of the generalized distributions for modelling life time data lies in the flexibility to model both monotonic and non-monotonic failure rates even though the baseline failure rate may be monotonic. The basic justification for generating a new distribution in practice are the following:

- to produce a skewness for symmetrical models;

- to define special models with all types of hrf;

- to construct heavy-tailed distributions for modelling various real data sets;

- to generate distributions with left-skewed, right-skewed, symmetric, or reversed-J shape;

- to provide consistently better fits than other generated distributions with the same underlying model;

The proposed distribution is clearly flexible to satisfy the above properties as demonstrated in Section 7. The rest of the paper is outlined as follows. In Section 2, we define three special models and provide the plots of their pdf's and hazard rate functions (hrf's). In Section 3, we derive some of its mathematical properties including ordinary and incomplete moments, generating functions, probability weighted moments (PWMs), residual life and reversed residual life functions, R'enyi, Shannon and q-entropies. Order statistics and their moments are investigated at the end of the section. Some characterizations results are pro-vided in Section 4. Maximum likelihood estimation of the model parameters is addressed in Section 5. Simple type Copula is constructed and a useful stochastic property is introduced in Section 6. In Section 7, we provide two applications to real data to illustrate the importance of the new family. In section 8 , simulation results to assess the performance of the proposed maximum likelihood estimation procedure are discussed. Finally, some concluding remarks are presented in Section 9. 


\section{Special Models}

We consider the following:

- Set $\mathrm{G}(\mathrm{x})=1-e^{-\mathrm{ax} \mathrm{x}^{b}}$, then $\mathrm{f}(\mathrm{x})$ will be GOGE-Weibull distribution.

- Set $\mathrm{G}(\mathrm{x})=\Phi(\mathrm{x} ; \mu, \sigma)$, then $\mathrm{f}(\mathrm{x})$ will be GOGE-Normal distribution, where $\Phi$ is cdf of the standard normal distribution

- Set $G(x)=1-\left(1-x^{a}\right)^{b}$, then $f(x)$ will be GOGE-Kumaraswamy distribution.

The plots of pdf and hrf for some special cases of GOGE-G are given in Figuers $1-4$.

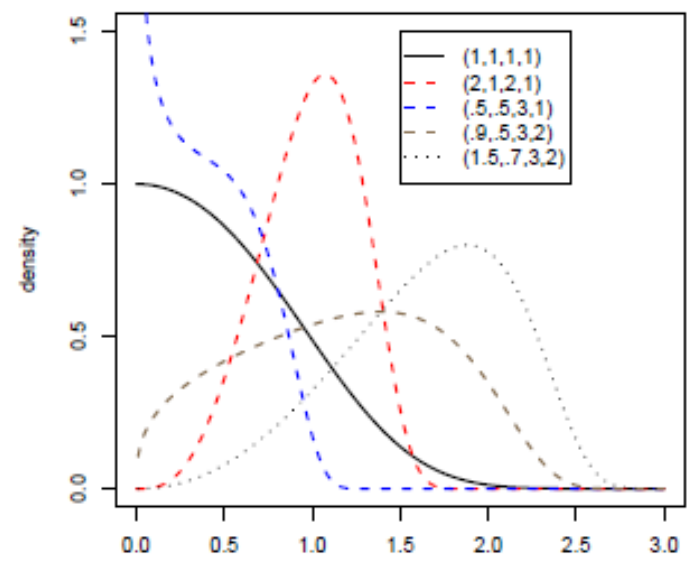

Figure 1: Plot of GOGE-Weibull( $(\alpha, \beta, \mathrm{a}, \mathrm{b})$ density for selected values of the parameters.

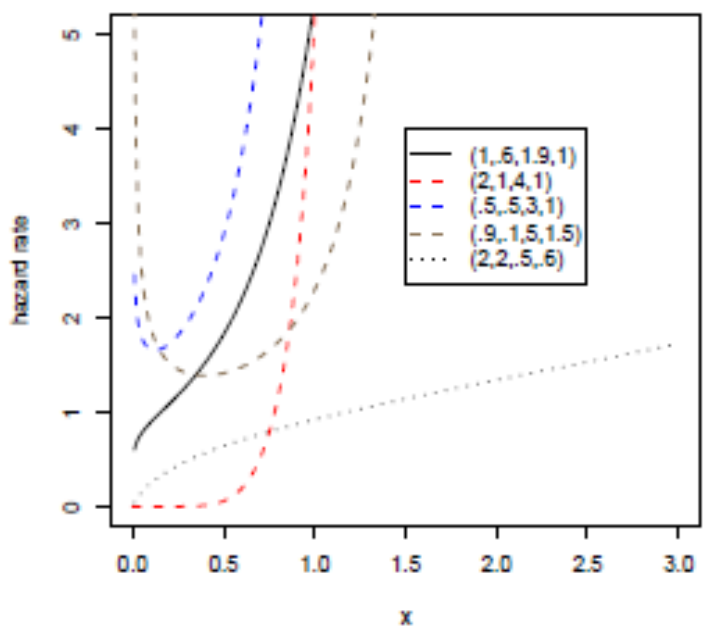

Figure 2: Plot of GOGE-Weibull $(\alpha, \beta, \mathrm{a}, \mathrm{b})$ hazard rate for selected values of the parameters. 


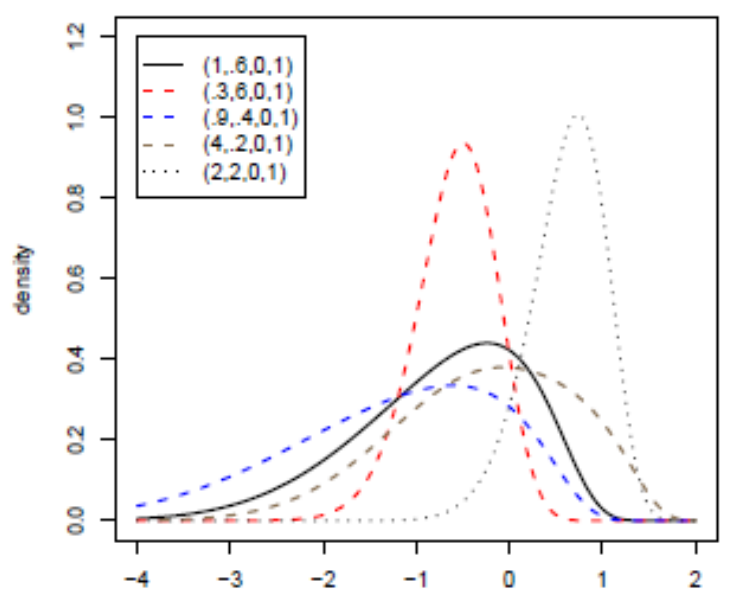

Figure 3: Plot of GOGE-Normal $(\alpha, \beta, \mu, \sigma)$ density for selected values of the parameters.

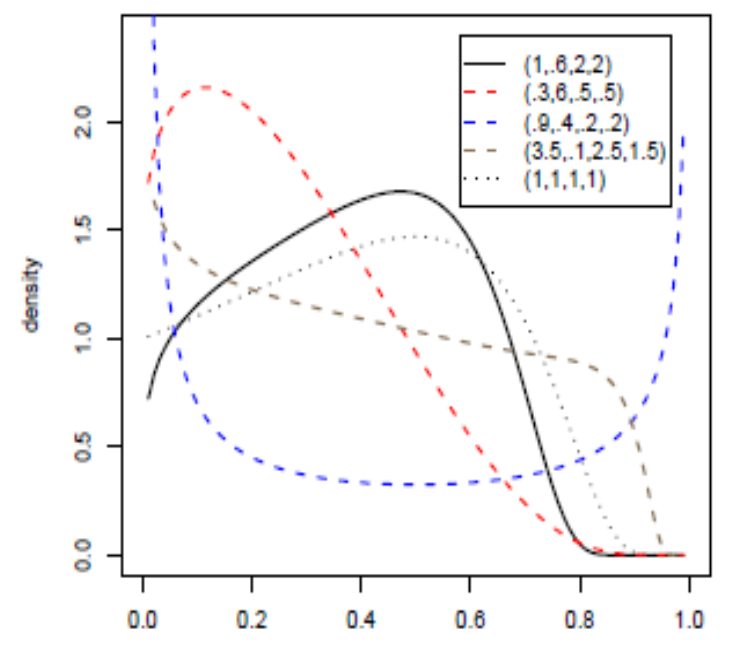

Figure 4: Plot of GOGE-Kumaraswamy $(\alpha, \beta, a, b)$ density for selected values of the parameters.

\section{Mathematical properties}

Here, we investigate mathematical properties of the GOGE-G family of distributions including ordinary moments, generating function, residual life and reversed residual life functions, entropies and order statistics.

\subsection{Moments and generating function}

The rth ordinary moment of $\mathrm{X}$ is given by $\mu_{r}^{\prime}=E\left(X^{r}\right)=\int_{-\infty}^{\infty} x^{r} f(x) d x$. Then we obtain

$$
\mu_{r}^{\prime}=\sum_{j, k=0}^{\infty} w_{j, k} E\left(Y_{\alpha(j+k+1)}^{r}\right),
$$

where, $Y \gamma$ denotes the exp-G distribution with power parameter $\gamma$. Setting $r=$ 1 in (6), we have the mean of $X$. The last integration can be computed numerically for most parent distributions. The skewness and kurtosis measures can be 
calculated from the ordinary moments using well-known relationships. The nth central moment of $\mathrm{X}$, say $\mathrm{M}_{\mathrm{n}}$, follows as

$$
M_{n}=E(X-\mu)^{n}=\sum_{h=0}^{n}(-1)^{h}\left(\begin{array}{l}
n \\
h
\end{array}\right)\left(\mu_{1}^{\prime}\right)^{n} \mu_{n-h}^{\prime} .
$$

The cumulants ( $\mathrm{k}_{\mathrm{n}}$ ) of X follow recursively from ${ }^{\kappa_{n}}=\mu_{n}^{\prime}-\sum_{r=0}^{n-1}\left(\begin{array}{c}n-1 \\ r-1\end{array}\right) \kappa_{r} \mu_{n-r}^{\prime}$; where $\kappa_{1}=\mu_{1}^{\prime}, \kappa_{2}=\mu_{2}^{\prime}-\mu_{1}^{\prime 2}, \kappa_{3}=\mu_{3}^{\prime}-3 \mu_{2}^{\prime} \mu_{1}^{\prime}+\mu_{1}^{\prime 3}$, etc. The skewness and kurtosis measures also can be calculated from the ordinary moments using well-known relationships. The moment generating function (mgf) $\mathrm{M}_{\mathrm{X}}(\mathrm{t})=\mathrm{E}\left(\mathrm{e}^{\mathrm{tX}}\right)$ of $\mathrm{X}$ can be derived from equation (4) as

$$
M_{X}(t)=\sum_{j, k=0}^{\infty} w_{j, k} M_{\alpha(j+k+1)}(t),
$$

where $M_{\delta}(t)$ is the mgf of $Y_{\delta}$. Hence, $M_{X}(t)$ can be determined from the mgfs of the exp-G distributions.

\subsection{Residual life and reversed residual life functions}

The nth moment of the residual life, say $m_{n}(t)=E[(X-t) n \mid X>t], n=1,2, \ldots$, uniquely determine $\mathrm{F}(\mathrm{x})$. The $\mathrm{nth}$ moment of the residual life of $\mathrm{X}$ is given by $m_{n}(t)=\frac{1}{R(t) \mid} \int_{t}^{\infty}(x-t)^{n} d F(x)$. Therefore

$$
m_{n}(t)=\frac{1}{R(t)} \sum_{j, k=0}^{\infty} w_{j, k}^{\star} \int_{t}^{\infty} x^{r} h_{\alpha(j+k+1)}(x),
$$

Where $w_{j, k}^{\star}=w_{j, k} \sum_{r=0}^{n}\left(\begin{array}{l}n \\ r\end{array}\right)(-t)^{n-r}$. Another interesting function is the mean residual life (MRL) function or the life expectation at age $t$ defined by $m_{1}(t)=E[(X$ $-\mathrm{t}) \mid X>t]$, which represents the expected additional life length for a unit which is alive at age $t$. The MRL of $X$ can be obtained by setting $n=1$ in the last equation. The $n$th moment of the reversed residual life, say $\operatorname{Mn}(\mathrm{t})=\mathrm{E}[(\mathrm{t}-\mathrm{X}) \mathrm{n} \mid \mathrm{X} \leq \mathrm{t}]$ for $\mathrm{t}$ $>0$ and $\mathrm{n}=1,2, \ldots$ uniquely determines $\mathrm{F}(\mathrm{x})$. We obtain $M_{n}(t)=\frac{1}{F(t)} \int_{0}^{t}(t-x)^{n} d F(x)$. Then, the nth moment of the reversed residual life of $\mathrm{X}$ becomes

$$
M_{n}(t)=\frac{1}{F(t)} \sum_{j, k=0}^{\infty} w_{j, k}^{\star \star} \int_{0}^{t} x^{r} h_{\alpha(j+k+1)}(x),
$$

where $w_{j, k}^{\star \star}=w_{j, k} \sum_{r=0}^{n}(-1)^{r}\left(\begin{array}{l}n \\ r\end{array} t^{n-r}\right.$. The mean inactivity time (MIT) or mean waiting time (MWT) also called the mean reversed residual life function is given by $\mathrm{M}_{1}(\mathrm{t})$ $=\mathrm{E}[(\mathrm{t}-\mathrm{X}) \mid \mathrm{X} \leq \mathrm{t}]$, and it represents the waiting time elapsed since the failure of an item on condition that this failure had occurred in $(0, t)$. The MIT of the GOGE$\mathrm{G}$ family of distributions can be obtained easily by setting $\mathrm{n}=1$ in the above equation.

\subsection{Entropies}

The Rényi entropy of a random variable $\mathrm{X}$ represents a measure of variation of the uncertainty. The Rényi entropy is defined by 


$$
I_{\theta}(X)=\frac{1}{1-\theta} \log \int_{-\infty}^{\infty} f(x)^{\theta} d x, \theta>0 \text { and } \theta \neq 1 .
$$

Using the pdf (6), we can write

$$
f(x)^{\theta}=\sum_{j, k=0}^{\infty} \Psi_{j, k} g(x)^{\theta} G(x)^{\alpha(j+k+\theta)-\theta},
$$

Where $\Psi_{j, k}=\frac{(\alpha \beta)^{\theta}(-1)^{j+k}\left(-\left(\begin{array}{c}(j+2) \\ k\end{array}\right)\right.}{j !} \sum_{i=0}^{\infty}(-1)^{i}(i+\theta)^{j}\left(\begin{array}{c}\theta(\beta-1) \\ i\end{array}\right)$. Then,the Rényi entropy of the GOGE-G family is given by

$$
I_{\theta}(X)=\frac{1}{1-\theta} \log \left\{\sum_{j, k=0}^{\infty} \Psi_{j, k} \int_{-\infty}^{\infty} g(x)^{\theta} G(x)^{\alpha(j+k+\theta)-\theta} d x\right\},
$$

The q-entropy, say $H q(X)$, can be obtained as

$$
H_{q}(X \mid)=\frac{1}{q-1} \log \left\{1-\left[\sum_{j, k=0}^{\infty} \Psi_{j, k}^{\star} \int_{-\infty}^{\infty} g(x)^{q} G(x)^{\alpha(j+k+q)-q} d x\right]\right\}
$$

where $\Psi_{j, k}^{\star}=\frac{(\alpha \beta)^{q}(-1)^{j+k}(-(j+2)}{j !} \sum_{i=0}^{\infty}(-1)^{i}(i+q)^{j}\left(\begin{array}{c}q(\beta-1) \\ i\end{array}\right) q>0, q \neq 1$.The Shan-non entropy of a random variable $\mathrm{X}$, say SI, is defined by $\mathrm{SI}=\mathrm{E}\{-[\log f(\mathrm{X})]\}$.

It is the special case of the $\mathrm{R}^{\prime}$ enyi entropy when $\theta \uparrow 1$.

\subsection{Order statistics}

Order statistics make their appearance in many areas of statistical theory and practice. Let $X_{1}, \ldots, X_{n}$ be a random sample from the GOGE-G family of distributions and let $\mathrm{X}_{1: \mathrm{n},}, \ldots, \mathrm{X}_{\mathrm{n}: \mathrm{n}}$ be the corresponding order statistics. The pdf of the ith order statistic, say $X_{i: n}$, can be written as

$$
f_{i: n}(x)=\frac{1}{\mathrm{~B}(i, n-i+1)} \sum_{j=0}^{n-i}(-1)^{j}\left(\begin{array}{c}
n-i \\
j
\end{array}\right) f(x) F^{j+i-1}(x),
$$

where $\mathrm{B}(\cdot, \cdot)$ is the beta function. Substituting (1) and (2) in equation (7) and using a power series expansion, we have

$$
\begin{array}{r}
f(x) F(x)^{j+i-1}=\sum_{w, k=0}^{\infty} d_{w, k} h_{\alpha(w+k+1)}(x), \\
\text { where } d_{w, k}=\frac{\alpha \beta(-1)^{w+k}\left(\begin{array}{l}
-(w+2) \\
k
\end{array}\right)}{w !(\alpha(w+m+1)]} \sum_{l=0}^{\infty}(-1)^{l}(l+1)^{w}\left(\begin{array}{c}
\beta(i+j)-1 \\
l
\end{array}\right) !
\end{array}
$$

can be expressed as

$$
f_{i: n}(x)=\sum_{j=0}^{n-i} \frac{(-1)^{j}\left(\begin{array}{c}
n-i \\
j
\end{array}\right)}{\mathrm{B}(i, n-i+1)} \sum_{w, k=0}^{\infty} d_{w, k} h_{\alpha(w+k+1)}(x) .
$$

The density function of the GOGE-G order statistics is a mixture of exp-G densities. Based on the last equation, we note that the properties of $X_{i: n}$ follow from those of $Y_{\alpha(w+k+1)}$. For example, the moments of $X_{i: n}$ can be expressed as

$$
E\left(X_{i: n}^{q}\right)=\sum_{w, k=0}^{\infty} \sum_{j=0}^{n-i} \frac{(-1)^{j}\left(\begin{array}{c}
n-i \\
j
\end{array}\right) d_{j, k}}{\mathrm{~B}(i, n-i+1)} E\left(Y_{\alpha(w+k+1)}^{q}\right) .
$$


The L-moments are analogous to the ordinary moments but can be estimated by linear combinations of order statistics. They exist whenever the mean of the distribution exists, even though some higher moments may not exist, and are relatively robust to the effects of outliers. Based upon the moments in equation (8), we can derive explicit expressions for the L-moments of $\mathrm{X}$ as infinite weighted linear combinations of the means of suitable GOGE-G order statistics.

\section{Characterizations}

In this section we present certain characterizations of GOGE-G distribution. The first characterization is based on hazard function and the second one is based on the conditional expectation of certain functions of the random variable.

\subsection{Characterization based on hazard function}

It is known that the hazard function, $\mathrm{h}_{\mathrm{F}}$, of a twice diff erentiable distribution function, $\mathrm{F}$, satisfies the first order diff erential equation

$$
\frac{f^{\prime}(x)}{f(x)}=\frac{h_{F}^{\prime}(x)}{h_{F}(x)}-h_{F}(x) .
$$

For many univariate continuous distributions, this is the only characterization available in terms of the hazard function. The following characterization establish a non-trivial characterization for GOGE-G distribution, with $\beta=1$, in terms of the hazard function, which is not of the above trivial form.

\section{Proposition 4.1.1.}

Let $\mathrm{X}: \Omega \rightarrow(0, \infty)$ be a continuous random variable. The $\mathrm{pdf}$ of $\mathrm{X}$ is $(6)$ if and only if its hazard function $\mathrm{hF}(\mathrm{x})$ satisfies the diff erential equation

$$
h_{F}^{\prime}(x)-\left(\frac{g^{\prime}(x ; \psi)}{g(x ; \psi)}\right) h_{F}(x)=\alpha(g(x ; \psi))^{2} G(x ; \psi)^{\alpha-2}\left\{\frac{(a-1)+2 \alpha G(x ; \psi)^{\alpha}}{\left[1-G(x ; \psi)^{a}\right]^{3}}\right\} .
$$

Proof. If X has pdf (6), then clearly the above diff erential equation holds. Now, if the diff erential equation holds, then

$$
\frac{d}{d x}\left\{\frac{1}{g(x ; \psi)} h_{F}(x)\right\}=\alpha \frac{d}{d x}\left\{\frac{G(x ; \psi)^{\alpha-1}}{\left[1-G(x ; \psi)^{a}\right]^{2}}\right\},
$$

or, equivalently,

$$
h_{F}(x)=\frac{\operatorname{ag}(x ; \psi) G(x ; \psi)^{\alpha-1}}{\left[1-G(x ; \psi)^{\alpha}\right]^{2}} .
$$

Integrating both sides of the above equation, we arrive at

$$
-\ln (1-F(x))=\frac{1}{1-G(x ; \psi)^{a}}-1=\frac{G(x ; \psi)^{a}}{1-G(x ; \psi)^{a}},
$$

or

$$
1-F(x)=\exp \left\{\frac{-G(x ; \psi)^{a}}{1-G(x ; \psi)^{a}}\right\}
$$




\subsection{Characterization based on conditional expectation}

In this subsection, we first establish a characterization of a distribution based on the conditional expectation of the random variable. Then we apply our characterization to the GOGE-G distribution.

\section{Proposition 4.2.1.}

Let $\mathrm{X}: \Omega \rightarrow(\mathrm{a}, \mathrm{b})$ be a continuous random variable with cdf $\mathrm{F}$ and corresponding pdf $f$. Let $\eta$ be a diff erentiable function and greater than 1 on $(a, b)$ such that $\lim _{x \rightarrow a^{+} \eta(x)}=1$ and $\lim _{x \rightarrow b^{-} \eta(x)}=1+c$. Then, for $0<c<1$,

$$
E[\eta(X) \mid X \leq x]=c+(1-c) \eta(x), \quad x \in(a, b),
$$

if and only if

$$
F(x)=\left(\frac{\eta(x)-1}{c}\right)^{\frac{1-c}{c}} .
$$

Proof. If (9) holds, then

$$
\int_{a}^{x} \eta(u) f(u) d u=\{c+(1-c) \eta(x)\} F(x) .
$$

Diff erentiating both sides of the above equation with respect to $\mathrm{x}$ and rearranging the terms, we arrive at

$$
\frac{f(x)}{F(x)}=\frac{1-c}{c}\left(\frac{\eta^{\prime}(x)}{\eta(x)-1}\right) .
$$

Integrating both sides of (11) from $\mathrm{x}$ to $\mathrm{b}$ and using the condition $\lim _{\mathrm{x} \rightarrow \mathrm{b}^{-} \eta(\mathrm{x})}=1+\mathrm{c}$, we arrive at (10). Conversely, if (10) holds, then $\psi(x)=1+c(F(x))^{\frac{c}{1-c}}$ and

$$
\begin{aligned}
E[\eta(X) \mid X \leq x] & =\frac{\int_{a}^{x}\left\{1+c(F(u))^{\frac{c}{1-c}}\right\} f(u) d u}{F(x)} \\
& =1+(1-c)(\eta(x)-1)=c+(1-c) \eta(x),
\end{aligned}
$$

which is (9).

\section{Remark 4.2.1.}

Taking $\eta(x)=c+1-\exp \left\{\frac{-G(x ; \psi)^{a}}{1-G(x ; \psi)^{a}}\right\},(a, b)=(0, \infty)$ and $\mathrm{c}=1+\beta$, Proposition 4.2.1 provides a characterization of GOGE-G distribution.

\section{Remark 4.2.2.}

Further special cases which can be characterized via proposition 4.2.1 with simple choices of $\eta(\mathrm{X})$ are:

1. $\mathrm{G}(\mathrm{x} ; \alpha, \psi)=\left(1-e^{-\psi x}\right)^{\frac{1}{\alpha}}, x>0$, for which $F(x)=\left[1-e^{1-e^{-\psi x}}\right]^{\beta}$.

2. $\mathrm{G}(\mathrm{x} ; \alpha)=x^{\frac{1}{\alpha}}, \quad 0 \leq \mathrm{x} \leq 1$, the power function distribution, for which $\mathrm{F}(\mathrm{x})=$ $\left[1-e^{1-(1-x)^{-1}}\right]^{\beta}$.

3. $\mathrm{G}(\mathrm{x})=\mathrm{x}$, the base line cdf is uniform on the unit interval, for which $\mathrm{F}(\mathrm{x})=$ $\left[1-e^{1-\left(1-x^{\alpha}\right)^{-1}}\right]^{\beta}$. 


\section{Estimation}

Several approaches for parameter estimation are proposed in the literature but the maximum likelihood method is the most commonly employed. The maxi-mum likelihood estimators (MLEs) enjoy desirable properties and can be used for constructing confidence intervals and regions and also in test statistics. The normal approximation for these estimators in large samples can be easily handled either analytically or numerically. So, we consider the estimation of the unknown parameters of this family from complete samples only by maximum likelihood method. Let $\mathrm{x}_{1}, \ldots, \mathrm{x}_{\mathrm{n}}$ be a random sample from the GOGE-G distribution with parameters $\alpha, \beta$ and $\psi$. Let $\Theta=\left(\alpha, \beta, \psi^{\top}\right)^{\top}$ be the $\mathrm{p} \times 1$ parameter vector. For determining the MLE of $\Theta$, we have the log-likelihood function

$$
\begin{aligned}
\ell= & \ell(\Theta)=n \log \alpha+n \log \beta+\sum_{i=1}^{n} \log g(x ; \psi)+(\alpha-1) \sum_{i=1}^{n} \log G(x ; \psi) \\
& -\sum_{i=1}^{n} s_{i}+(\beta-1) \sum_{i=1}^{n} \log \left(1-e^{-s_{i}}\right)-2 \sum_{i=1}^{n} \log z_{i},
\end{aligned}
$$

where $s_{i}=\frac{G\left(x_{i} ; \psi^{\alpha}\right.}{z_{i}}$ and $z_{i}=1-G\left(x_{i} ; \psi\right)^{\alpha}$. The components of the score vector, $\mathbf{U}(\Theta)=\frac{\partial \ell}{\partial \Theta}=\left(\frac{\partial \ell}{\partial \alpha}, \frac{\partial \ell}{\partial \beta}, \frac{\partial \ell}{\partial \psi}\right)^{\top}$, are

$$
\begin{gathered}
U_{\alpha}=\frac{n}{\alpha}+\sum_{i=1}^{n} \log G(x ; \psi)-\sum_{i=1}^{n} w_{i}+(\beta-1) \sum_{i=1}^{n} \frac{w_{i} e^{-s_{i}}}{1-e^{-s_{i}}}-2 \sum_{i=1}^{n} \frac{p_{i}}{z_{i}}, \\
U_{\beta}=\frac{n}{\beta}+\sum_{i=1}^{n} \log \left(1-e^{-s_{i}}\right)
\end{gathered}
$$

and

$$
U_{\psi}=\sum_{i=1}^{n} \frac{g^{\prime}\left(x_{i} ; \psi\right)}{g\left(x_{i} ; \psi\right)}+(\alpha-1) \sum_{i=1}^{n} \frac{G^{\prime}\left(x_{i} ; \psi\right)}{G\left(x_{i} ; \psi\right)}-\sum_{i=1}^{n} m_{i}+(\beta-1) \sum_{i=1}^{n} \frac{m_{i} e^{-s_{i}}}{1-e^{-s_{i}}}-2 \sum_{i=1}^{n} \frac{q_{i}}{z_{i}},
$$

where $g^{\prime}\left(x_{i} ; \psi\right)=\partial g\left(x_{i} ; \psi\right) / \partial \psi, G^{\prime}\left(x_{i} ; \psi\right)=\partial G\left(x_{i} ; \psi\right) / \partial \psi, w_{i}=\frac{G\left(x_{i} ; \psi\right)^{\alpha} \log G\left(x_{i} ; \psi\right)}{\left[1-G\left(x_{i} ; \psi\right)^{\alpha}\right]^{2}}$ and $\mathrm{m}_{\mathrm{i}}=\frac{\alpha G^{\prime}\left(x_{i} ; \psi\right) G\left(x_{i} ; \psi\right)^{\alpha-1}}{\left[1-G\left(x_{i} ; \psi\right)^{\alpha}\right]^{2}}$. Setting the nonlinear system of equations $\mathrm{U}_{\alpha}=\mathrm{U}_{\beta}=0$ and $\mathrm{U} \psi=0$ and solving them simultaneously yields the $\operatorname{MLE} \widehat{\Theta}=\left(\widehat{\alpha}, \widehat{\beta}, \widehat{\psi}^{\top}\right)^{\top}$. To solve these equations, it is usually more convenient to use nonlinear optimization methods such as the quasi-Newton algorithm to numerically maximize $\ell$. For interval estimation of the parameters, we obtain the $\mathrm{p} \times \mathrm{p}$ observed information matrix $J(\Theta)=\left\{\frac{\partial^{2} \ell}{\partial r \partial s}\right\}$ (for $\left.\mathrm{r}, \mathrm{s}=\alpha, \beta, \psi\right)$, whose elements can be computed numerically. Under standard regularity conditions when $n \rightarrow \infty$, the distribution of $\widehat{\Theta}$ can be approximated by a multivariate normal $\mathrm{Np}(0, \mathrm{~J}(\widehat{\Theta})-1)$ distribution to construct approximate confidence intervals for the parameters. Here, $J(\widehat{\Theta})$ is the total observed information matrix evaluated at $\widehat{\Theta}$. The method of the re-sampling bootstrap can be used for correcting the biases of the MLEs of the model parameters. Good interval estimates may also be obtained using the boot-strap percentile method. 


\section{Simple type Copula based construction}

We consider several different approaches to construct bivariate and multivariate GOGE-G type distributions via copula (or even with straightforward bivariate cdf functions form, in which we just need to consider two diff erent GOGE-G cdfs). It must be mentioned here that in all our derivations, we have considered the same set of parameters $\psi$ for the baseline cdf G. So, these derived models, depend a lot on the behavior of the baseline cdf.

\subsection{Via Morgenstern family}

First, we start with cdf for Morgenstern family of two random variables $\left(\mathrm{X}_{1}, \mathrm{X}_{2}\right)$ which has the following form:

$$
F\left(x_{1}, x_{2}\right)=G_{1}\left(x_{1}\right) G_{2}\left(x_{2}\right)\left\{1+\lambda\left(1-G_{1}\left(x_{1}\right)\right)\left(1-G_{2}\left(x_{2}\right)\right)\right\},
$$

where $|\lambda| \leq 1$. Now, if we set the following:

$$
\begin{aligned}
& \text { - } G_{1}\left(x_{1}\right)=F\left(x_{1}\right)=\left[1-e^{\frac{-G\left(x_{1} ; \psi\right)^{\alpha}}{1-G(x ; \psi)^{\alpha}}}\right]^{\beta_{1}}, \\
& \text { - } G_{2}\left(x_{2}\right)=F\left(x_{2}\right)=\left[1-e^{\frac{-G\left(x_{2} ; \psi\right)^{\alpha}}{1-G(x ; \psi)^{\alpha}}}\right]^{\beta_{2}},
\end{aligned}
$$

then we have a $(5+\psi)$ dimension parameter model. Needless to say, the estimation will be a big issue here. Estimation via Bayesian paradigm may be done, but again, the choice of appropriate priors will be challenging.

\subsection{Via several type existing Copula models}

We consider the following inequel:

- Via Clayton copula: Some might claim that this is a weighted version of the Clayton copula, which is of the form:

$$
C(u, v)=\left[u^{-\left(\delta_{1}+\delta_{2}\right)}+v^{-\left(\delta_{1}+\delta_{2}\right)}-1\right]^{-1 /\left(\delta_{1}+\delta_{2}\right)} .
$$

This is indeed a valid copula. Next, let us assume that $\mathrm{X} \sim \mathrm{GOGE}-\mathrm{G}\left(\alpha_{1}, \beta_{1}, \psi\right)$ and $\mathrm{Y} \sim \mathrm{GOGE}-\mathrm{G}\left(\alpha_{2}, \beta_{2}, \psi\right)$. Then, setting $\mathrm{u}=\left[1-e^{\frac{-G(x ; \psi) \alpha_{1}}{1-G(x ; \psi) \alpha_{1}}}\right]^{\beta_{1}}$ and $\mathrm{v}=\mathrm{F}(\mathrm{y})=$ $\left[1-e^{\frac{-G(y ; \psi)^{\alpha_{2}}}{1-G(y ; \psi)^{\alpha 2}}}\right]^{\beta_{2}}$,

(the cdf)

$$
H(x, y)=\left\{\left[1-e^{\frac{-G(x ; \psi)^{\alpha_{1}}}{1-G(x ; \psi)^{\alpha_{1}}}}\right]^{-\beta_{1}\left(\delta_{1}+\delta_{2}\right)}+\left[1-e^{\frac{-G(y ; \psi)^{\alpha}}{1-G(y ; \psi)^{\alpha_{2}}}}\right]^{-\beta_{2}\left(\delta_{1}+\delta_{2}\right)}-1\right\}^{-1 /\left(\delta_{1}+\delta_{2}\right)} .
$$

Note: Depending on the specific baseline cdf, one may construct various bivariate GOGE-G type models in which $\left(\delta_{1}+\delta_{2}\right) \geq 0$.

Multivariate extension: A straightforward d-dimensional extension from the above will be 


$$
H\left(x_{1}, x_{2}, \cdots, x_{d}\right)=\left[\sum_{i=1}^{d}\left\{1-e^{\frac{-G\left(x_{i} ;\right)^{\alpha_{i}}}{1-G\left(x_{i} ; \psi\right)^{\alpha_{i}}}}\right\}^{-\beta_{i}\left(\delta_{1}+\delta_{2}\right)}-d+1\right]^{-1 /\left(\delta_{1}+\delta_{2}\right)} .
$$

- Via a special type of Copula to start with this type of copula, first we will mention one theorem and the corresponding corollary.

Theorem 6.2.1: Let $\mathrm{F}_{\mathrm{i}}, \mathrm{i}=1, \ldots, \mathrm{n}$ be absolutely continuous one dimensional marginal cumulative distribution functions with domain $I$ of an absolutely continuous $\mathrm{n}$-dimensional cumulative distribution $\mathrm{F}$ with domain In. If $\mathrm{F}$ maximizes Renyi's entropy by rescaling (or maiming Tsallis entropy) when the entropy index equals 2 , then $\mathrm{F}$ will be

$$
\mathrm{F}\left(x_{1}, \ldots, x_{n}\right)=\sum_{i=1}^{n} F_{i}\left(x_{i}\right) \prod_{j=1}^{n} x_{j}+(1-n) \prod_{i=1}^{n} x_{i}
$$

Note that $\mathrm{I}=[0,1]$. For the proof in the bivariate case and the extension to the multivariate case see Pougaza et al. (2011).

Corollary 6.2.1: Let $\mathrm{F}_{\mathrm{i}}$, for $\mathrm{i}=1,2, \cdots, \mathrm{n}$ be as in Theorem 6.2.1, and let $F_{i}^{-1}$ be quasiinverse (see page 21 of Nelsen, R.B. (1999)) of $F_{i}$.

Then, for any $\mathrm{u}$ in $\mathrm{I}^{\mathrm{n}}$,

$$
C\left(u_{1}, \cdots, u_{n}\right)=\max \left(\sum_{i=1}^{n} u_{i} \prod_{j=1(\neq i)}^{n} F_{j}^{(-1)}\left(u_{j}\right)+(1-n) \prod_{i=1}^{n} F_{i}^{(-1)}\left(u_{i}\right), 0\right)
$$

is a copula. It appears that one can use Theorem 6.2.1 as a constructive method for determining a joint multivariate distribution from the only knowledge of its marginals and the Corollary 6.2.1 as a tool for creating families of copula just by specifying marginals. In the bivariate case, we have $\mathrm{C}(\mathrm{u}, \mathrm{v})=\mathrm{v} \phi(\mathrm{u})+\mathrm{u} \delta(\mathrm{v})-\phi(\mathrm{u}) \delta(\mathrm{v})$, where $F_{1}^{-1}\left(\mathrm{u}_{1}\right) \equiv \phi$ and $F_{2}^{-1}\left(\mathrm{u}_{2}\right) \equiv \delta$, for any univariate functions $\phi$ and $\delta$, with the following properties:

$$
\begin{aligned}
& \text { 1. } \phi(0)=\delta(0)=0, \\
& \text { 2. } \phi(1)=\delta(1)=1,
\end{aligned}
$$

where $\phi, \delta$ are absolutely continuous distributions on $[0,1]$ and their derivatives are bounded for almost every values taken in $[0,1]$. Now, from our GOGE-G cdf, we can write, for any $\mathrm{u} \in(0,1)$,

$$
x=F^{-1}(u)=G^{-1}\left[\left[1-\left(1+\log \left(1 /\left(1-u^{1 / \beta}\right)\right)\right)^{-1}\right]^{1 / \alpha}\right] .
$$

So, a reasonable choice of bivariate copula for GOGE-G model may be considered as follows:

$$
\begin{aligned}
& C\left(u_{1}, u_{2}\right) \\
& =u_{1}\left[G^{-1}\left[\left\{1-\left(1+\log \left(1 /\left(1-u_{2}^{1 / \beta_{2}}\right)\right)\right)^{-1}\right\}^{1 / \alpha_{2}}\right]\right] \\
& +u_{2}\left[G^{-1}\left[\left\{1-\left(1+\log \left(1 /\left(1-u_{1}^{1 / \beta_{1}}\right)\right)\right)^{-1}\right\}^{1 / \alpha_{1}}\right]\right] \\
& -\left(\left[G^{-1}\left[\left\{1-\left(1+\log \left(1 /\left(1-u_{2}^{1 / \beta_{2}}\right)\right)\right)^{-1}\right\}^{1 / \alpha_{2}}\right]\right]\right) \\
& \times\left(\left[G^{-1}\left[\left\{1-\left(1+\log \left(1 /\left(1-u_{1}^{1 / \beta_{1}}\right)\right)\right)^{-1}\right\}^{1 / \alpha_{1}}\right]\right]\right) .
\end{aligned}
$$

If $\mathrm{G}$ is a proper cdf, then $\mathrm{C}\left(\mathrm{u}_{1}, \mathrm{u}_{2}\right)$ will be a valid copula. Assuming $\mathrm{G}$ is a valid cdf, one can easily verify the following: 


$$
\begin{aligned}
& -C(0,0)=1 . \\
& -C(1,1)=1 . \\
& \text { - } C\left(u_{1}, 0\right)=C\left(0, u_{2}\right)=0 . \\
& \text { - } C(t, 1)=C(1, t)=t . \\
& \text { - } \frac{\partial}{\partial u_{1}} C\left(u_{1}, u_{2}\right) \geq 0 \text { and } \frac{\partial}{\partial u_{2}} C\left(u_{1}, u_{2}\right) \geq 0 .
\end{aligned}
$$

Hence, it is indeed a valid bivariate GOGE-G type copula. Hence, the associated bivariate cdf (using Theorem 6.2.1) will be

$$
\begin{aligned}
F\left(x_{1}, x_{2}\right) & =x_{2} F_{1}\left(x_{1}\right)+x_{1} F_{2}\left(x_{2}\right)-x_{1} x_{2} \\
& =x_{2}\left[1-e^{\frac{-G\left(x_{1} ; \psi\right)^{\alpha_{1}}}{1-G\left(x_{1} ; \psi\right)^{\alpha_{1}}}}\right]^{\beta_{1}}+x_{1}\left[1-e^{\frac{-G\left(x_{2} ; \psi\right)^{\alpha_{2}}}{1-G\left(x_{2} ; \psi\right)^{\alpha}}}\right]^{\beta_{2}}-x_{1} x_{2} .
\end{aligned}
$$

Let's just call this bivariate GOGE-G copula (Type I). One may obtain the multivariate extension of the following form:

1. Multivariate GOGE-G copula (Type I)

$$
C(\vec{u})=\sum_{i=1}^{k}\left[\theta_{i} u_{i} \prod_{j_{1} \neq i} G^{-1}\left(A\left(u_{j_{1}}\right)\right]+\left(1-\sum_{i=1}^{k} \theta_{i}\right) u_{i}\left[\prod_{j_{1} \neq i} G^{-1}\left(A\left(u_{j_{1}}\right)\right],\right.\right.
$$

where $A\left(u_{j_{1}}\right)=\left\{1-\left(1+\log \left(1 /\left(1-u_{j_{1}}^{1 / \beta}\right)\right)\right)^{-1}\right\}^{1 / \alpha},($ assuming fixed $\alpha$ and $\beta$.)

2. Multivariate GOGE-G copula (Type II, based on bivariate F-G-M copula)

$$
C(\vec{u})=\prod_{i=1}^{k} u_{i}\left[1+\delta \prod_{i=1}^{k} G^{-1}\left(A\left(u_{i}\right)\right],\right.
$$

where $A($.$) is defined earlier and |\delta| \leq 1$.

\subsection{Stochastic property}

Theorem 6.3.1: Suppose $X_{1} \sim \mathrm{GOEG}-\mathrm{G}\left(\alpha_{1}, \beta_{1}\right)$ and $\mathrm{X}_{2} \sim \mathrm{GOEG}-\mathrm{G}\left(\alpha_{2}, \beta_{2}\right)$. Then $\mathrm{X}_{1}$ is stochastically smaller than $X_{2}$ if $\alpha_{1}>\alpha_{2}$ and $\beta_{1}>\beta_{2}$.

Note that for any $\alpha_{1}>\alpha_{2}$

$$
G(t ; \psi)^{\alpha_{1}}>G(t ; \psi)^{\alpha_{2}} .
$$

This is true for both integer and fractional values of $\alpha_{1}$ and $\alpha_{2}$. After some algebra, we get the following:

Since for $\alpha_{1}>\alpha_{2}$

$$
\begin{gathered}
\{G(t ; \psi)\}^{\alpha_{1}}>\{G(t ; \psi)\}^{\alpha_{2}} \\
{\left[1-G^{\alpha_{1}}(t ; \psi)\right]<\left[1-G^{\alpha_{2}}(t ; \psi)\right]} \\
\frac{\{G(t ; \psi)\}^{\alpha_{1}}}{1-G^{\alpha_{1}}(t ; \psi)}>\frac{\{G(t ; \psi)\}^{\alpha_{2}}}{1-G^{\alpha_{2}}(t ; \psi)} \\
\frac{-\{G(t ; \psi)\}^{\alpha_{1}}}{1-G^{\alpha_{1}}(t ; \psi)}<\frac{-\{G(t ; \psi)\}^{\alpha_{2}}}{1-G^{\alpha_{2}}(t ; \psi)}
\end{gathered}
$$

Rest of the proof follows immediately from here 


$$
\begin{gathered}
-\frac{G(t ; \psi)^{\alpha_{1}}}{1-G(t ; \psi)^{\alpha_{1}}}<-\frac{G(t ; \psi)^{\alpha_{2}}}{1-G(t ; \psi)^{\alpha_{2}}} \\
\rightarrow\left\{1-e^{\left[\frac{-G(x ; \psi)^{\alpha_{1}}}{1-G(x ; \psi)^{\alpha_{1}}}\right]}\right\}^{\beta_{1}}>\left\{1-e^{\left[\frac{-G(x ; \psi)^{\alpha_{2}}}{1-G(x ; \psi)^{\alpha_{2}}}\right]}\right\}^{\beta_{2}} \\
\rightarrow 1-\left\{1-e^{\left[\frac{-G(x ; \psi)^{\alpha_{1}}}{1-G(x ; \psi)^{\alpha_{1}}}\right]}\right\}^{\beta_{1}}<1-\left\{1-e^{\left[\frac{-G(x ; \psi)^{\alpha_{2}}}{1-G(x ; \psi)^{\alpha_{2}}}\right]}\right\}^{\beta_{2}} .
\end{gathered}
$$

This completes the proof.

\section{Applications}

In this section, we provide two applications to illustrate the importance of the GOGEW and GOGEE distributions presented in Section 2. The MLEs of the model parameters are computed and goodness-of-fit statistics for these distributions are compared with other competing distributions. The first real data set is a subset of data reported by Bekker et al. (2000) which corresponds to the survival times (in years) of a group of patients given chemotherapy treatment alone. The second real data set was originally reported by Nadarajah and Kotz (2007), which represents the fracture toughness MPa $\mathrm{m} 1 / 2$ data from the mate-rial Alumina. In the applications, the information about the hazard shape can help in selecting a particular model. For this porpose, a device called the total time on test (TTT) plot (Aarset, 1987) is useful. The TTT plot is obtained by plotting $G(r / n)=\left(\left(\sum_{i=1}^{r} y_{(i)}\right)+(n-r) y_{(r)}\right) / \sum_{i=1}^{n} y_{(i)}$ where $r=1, \ldots, n \quad$ and $y_{(i)} \quad(i=1, \ldots, n)$ are the order statistics of the sample, against $\mathrm{r} / \mathrm{n}$. If the shape is a straight diagonal, the hazard is constant. It is convex shape for decreasing hazards and concave shape for increasing hazards. The bathtub-shaped hazard is obtained when the first convex and then concave and for bimodal shape hazard, the TTT plot is first concave and then convex. The TTT plot for both datasets presented in Figure 2. These figures indicate that first and second dataset have constant and increasing failure rate functions.

In the first application, we shall compare the GOGEW model with other comparative models: the beta Weibull (BW) (Famoye et al., 2005), the Kumaraswamy Weibull (KwW) (Cordeiro et al., 2010), the exponentiated Weibull (EW) (Mud-holkar et al., 1993) and the Weibull Geometric (WG) (Barreto-Souza et al., 2011) distributions. In the second application, we compare the GOGEE model with other comparative models: the beta-exponential(BE) (Nadarajah and Kotz, 2006), the gamma exponentiated exponential (GEE) (Ristic and Balakrishnan, 2012), exponentiated exponential geometric (EEG) (Louzada et al., 2012) and the exponentiated exponential Poisson (EEP)( Ristic and Nadarajah, 2012) models. The MLEs are computed using the Limited-Memory Quasi-Newton Code for Bound-Constrained Optimization (L-BFGS-B) as well as the measures of goodness-of-fit including the log-likelihood function evaluated at the MLEs. The measures of goodness-of-fit including the Akaike information criterion (AIC), consistent Akaike information criterion (CAIC) (Burnham and Anderson, 2002), Hannan-Quinn information criterion (HQIC), Anderson-Darling ( $\mathrm{A}^{*}$ ), Cramer-von Mises $\left(\mathrm{W}^{*}\right)$ statistics are computed to compare the fitted models. The statistics $\mathrm{A}^{*}$ 
and $\mathrm{W}^{*}$ are described in Evans et al. (2008). They showed $\mathrm{W}^{*}$ and $\mathrm{A}^{*}$ can be calculated as

$$
W^{*}=\sum_{i=1}^{n}\left(\hat{F}\left(x_{(i)}\right)-\frac{i-0.5}{n}\right)^{2}+\frac{1}{12 n}
$$

And

$$
A^{*}=-\sum_{i=1}^{n} \frac{2 i-1}{n}\left(\ln \left(\hat{F}\left(x_{(i)}\right)\right)+\ln \left(1-\hat{F}\left(x_{(n+1-i)}\right)\right)\right)-n .
$$

In general, the smaller values of these statistics show the better fit to the data sets. The numerical values of the AIC, AICc, HQIC, $\mathrm{A}^{*}$ and $\mathrm{W}^{*}$ statistics are listed in Tables 5 and 6, whereas Tables 3 and 4 list the MLEs and their corresponding standard errors (in parentheses) of the model parameters. In Table 3, we compare the fits of the GOGEW distribution with the BW, KW, WG and EW models. We note that the GOGEW model gives the lowest values for the AIC, AICc, HQIC, A* and $\mathrm{W}^{*}$ statistics (for the survival times of cancer patients data) among the fitted models. So, the GOGEW distribution could be chosen as the best distribution. The emprical cdf (each dot show a step of it) with fitted GOGEW and rivals cdfs (curves plot on the dots) are displayed in Figure 3. In Table 4, we compare the fits of the GOGEE distribution with the BE, GEE, EEG and EEP distributions. We note that the GOGEE distribution gives the lowest values for the AIC, AICc, HQIC, A* and $\mathrm{W}^{*}$ statistics (for the fracture toughness data) among all fitted distributions. So, the GOGEE model can be chosen as the best model. The emprical cdf (each dot shows a step of it) with fitted GOGEE and rivals cdfs (curves plot on the dots) are displayed in Figure 4. It is very clear from Tables 3 and 4, and Figures 6 and 7 that the GOGEW and GOGEE models provide the best fits to the histogram of these data sets.
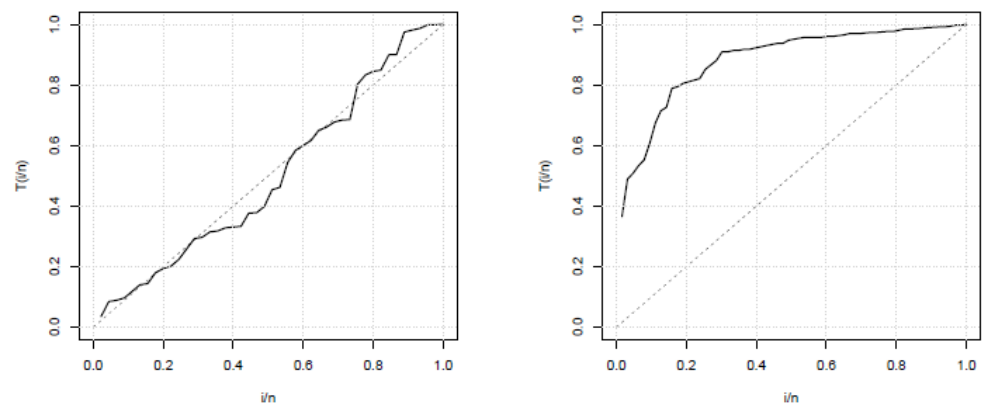

Figure 5: TTT-plot for the first dataset (left figure) and for the second dataset (right figure).

Table 1: Parameters estimates and standard deviation in parenthesis for first dataset

\begin{tabular}{|l|llll|}
\hline Model & \multicolumn{3}{|c|}{ Estimates } & (Standard deviation) \\
\hline & $\hat{\alpha}$ & $\hat{\beta}$ & $\hat{\lambda}$ & $\hat{c}$ \\
\hline GOGEW & $22.372(2.172)$ & $0.191(0.028)$ & $0.479(0.023)$ & $0.234(0.029)$ \\
\hline$B W$ & $2.012(0.213)$ & $5.121(0.584)$ & $0.196(0.029)$ & $0.697(0.059)$ \\
\hline & $\hat{a}$ & $\hat{b}$ & $\hat{\lambda}$ & $\hat{c}$ \\
\hline$K w W$ & $11.575(0.481)$ & $40.600(6.052)$ & $3.273(0.502)$ & $0.177(0.012)$ \\
\hline & $\hat{\alpha}$ & $\hat{\lambda}$ & $\hat{c}$ & \\
\hline$E W$ & $1.612(0.240)$ & $1.155(1.173)$ & $0.807(0.081)$ & \\
\hline & $\hat{p}$ & $\hat{\lambda}$ & $\hat{c}$ & \\
\hline$W G$ & $0.556(0.118)$ & $0.489(0.072)$ & $1.232(0.137)$ & \\
\hline
\end{tabular}


Figure 8 shows that GOGEW distribution is a good model for right skew heavy tail data sets and GOGEE distribution is suitable model for Leptokurtic data sets. The GOGEE can better model data sets with wider range of kurtosis among other generalized exponential distributions.

Table 2: Parameters estimates, standard deviation in parenthesis and log-likelihood for second dataset

\begin{tabular}{|c|c|c|c|}
\hline Model & & Estimates & (Standard deviation) \\
\hline & $\hat{\alpha}$ & $\hat{\beta}$ & $\hat{\lambda}$ \\
\hline \multirow[t]{2}{*}{ GOGEE } & $7.060(0.384)$ & $1.701(0.155)$ & $0.564(0.011)$ \\
\hline & $\hat{a}$ & $\hat{b}$ & $\hat{\lambda}$ \\
\hline \multirow[t]{2}{*}{$B E$} & $15.628(0.380)$ & $108.398(2.677)$ & $0.031\left(7 e^{-4}\right)$ \\
\hline & $\hat{\lambda}$ & $\hat{\alpha}$ & $\hat{\delta}$ \\
\hline \multirow[t]{2}{*}{ GEE } & $0.151(0.003)$ & $22.013(0.492)$ & $16.784(0.369)$ \\
\hline & $\hat{\alpha}$ & $\hat{\theta}$ & $\hat{\lambda}$ \\
\hline \multirow[t]{2}{*}{$E E G$} & $30.651(2.809)$ & $0.999(0.141)$ & $0.909(0.025)$ \\
\hline & $\hat{\alpha}$ & $\hat{\beta}$ & $\hat{\lambda}$ \\
\hline EEP & $30.615(2.803)$ & $0.908(0.025)$ & $0.010(0.317)$ \\
\hline
\end{tabular}

Table 3: Formal goodness-of-fit tests for the first data set

\begin{tabular}{|l|l|l|l|l|l|}
\hline Model & $A I C$ & $H Q I C$ & $C A I C$ & $A^{*}$ & $W^{*}$ \\
\hline $\begin{array}{l}\text { GOGEW } \\
(\alpha, \beta, \lambda, c)\end{array}$ & $\mathbf{1 2 0 . 3 7 3}$ & $\mathbf{1 2 3 . 0 6 7}$ & $\mathbf{1 2 1 . 3 7 3}$ & $\mathbf{0 . 4 2 9}$ & $\mathbf{0 . 0 5 8}$ \\
\hline $\begin{array}{l}B W \\
(\alpha, \beta, \lambda, c)\end{array}$ & 123.992 & 126.686 & 124.992 & 0.472 & 0.070 \\
\hline $\begin{array}{l}K w W \\
(a, b, \lambda, c)\end{array}$ & 124.130 & 126.824 & 125.130 & 0.468 & 0.070 \\
\hline $\begin{array}{l}E W \\
(\alpha, \lambda, c)\end{array}$ & 122.087 & 124.107 & 122.672 & 5.205 & 0.734 \\
\hline $\begin{array}{l}\text { MOW } \\
(p, \lambda, c)\end{array}$ & 121.618 & 123.639 & 122.204 & 0.490 & 0.071 \\
\hline
\end{tabular}

GOGEW

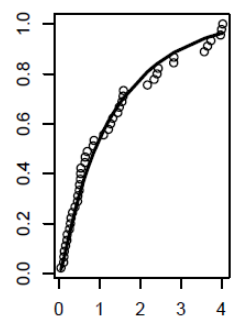

EW

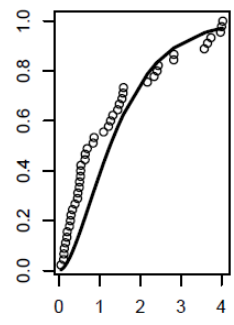

$\mathrm{x}$
BW

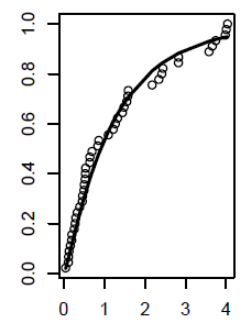

WG

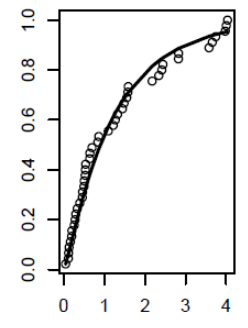

$\mathrm{x}$

Figure 6: Emprical cdf with cdf of fitted distributions for the first data set. 
Table 4: Formal goodness-of-fit tests for the second data set

\begin{tabular}{|l|l|l|l|l|l|}
\hline Model & $A I C$ & $H Q I C$ & $C A I C$ & $A^{*}$ & $W^{*}$ \\
\hline $\begin{array}{l}G O G E E \\
(\alpha, \beta, \lambda)\end{array}$ & $\mathbf{3 4 6 . 5 5}$ & $\mathbf{3 4 9 . 9 4}$ & $\mathbf{3 4 6 . 7 6}$ & $\mathbf{1 . 0 5 8}$ & $\mathbf{0 . 1 8 5}$ \\
\hline $\begin{array}{l}B E \\
(a, b, \lambda)\end{array}$ & 360.76 & 364.14 & 360.97 & 2.331 & 0.384 \\
\hline $\begin{array}{l}G E E \\
(\lambda, \alpha, \delta)\end{array}$ & 362.18 & 365.57 & 362.39 & 2.480 & 0.412 \\
\hline $\begin{array}{l}E E G \\
(\alpha, \theta, \lambda)\end{array}$ & 380.85 & 384.24 & 381.06 & 4.224 & 0.718 \\
\hline $\begin{array}{l}E E P \\
(\alpha, \beta, \lambda)\end{array}$ & 380.90 & 384.28 & 381.11 & 4.226 & 0.718 \\
\hline
\end{tabular}
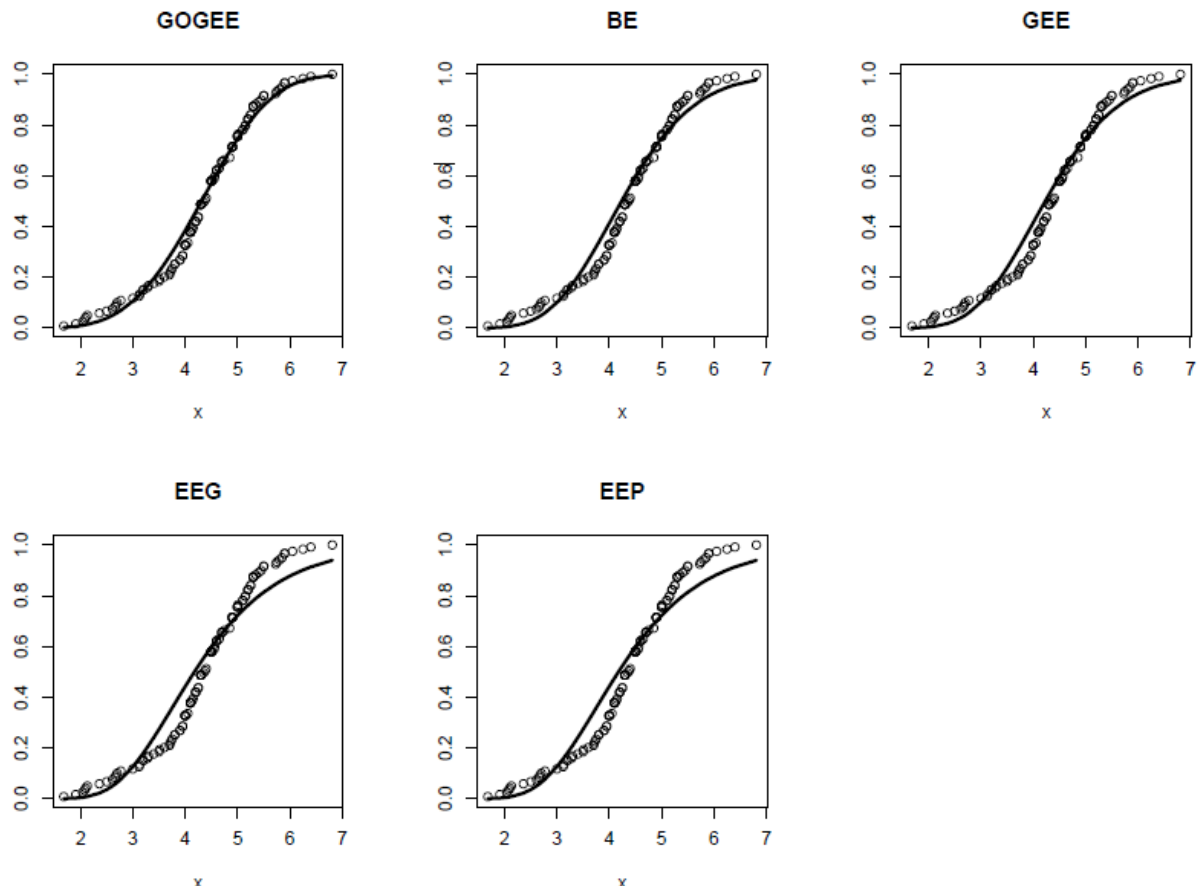

Figure 7: Emprical cdf with cdf of fitted distributions for the second data set.
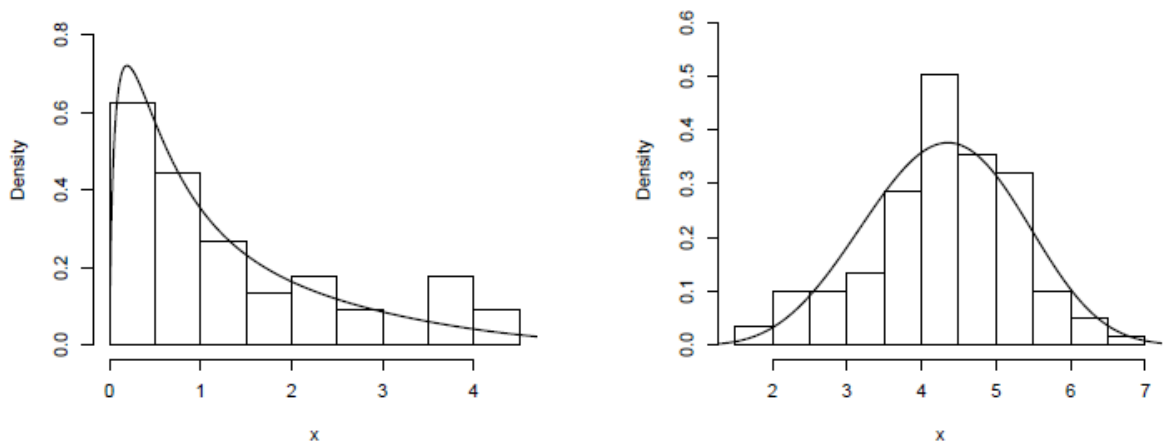

Figure 8: Fitted special cases of proposed model on histogram of data:(left) first data set, (right) second edata set. 


\section{Simulation study}

In this section, we consider the maximum likelihood estimation of parameters for the two models derived in the preceding sections. The maximum likelihood estimators can be obtained by direct maximization of the likelihood functions given below. In simulations and real life data applications described later on, we maximized the log-likelihood function using SAS PROC NLMIXED. For each maximization, the SAS PROC NLMIXED function was executed for a wide range of initial values and the maximum likelihood estimates were determined as the ones that corresponds to the largest of the maxima.

\subsection{Simulation study for GOGE-Normal distribution}

In this case, the log likelihood function is given by (from (2)

$$
\begin{aligned}
\ell= & n\left(\log \alpha+\log \beta-\frac{1}{2} \log \pi-\log \sigma\right)-\sum_{i=1}^{n} \log \left(\frac{x_{i}-\mu}{\sigma}\right) \\
& +(\alpha-1) \sum_{i=1}^{n} \log \left(\Phi\left[\frac{x_{i}-\mu}{\sigma}\right]\right)-\frac{1}{2} \sum_{i=1}^{n} \log \left\{1-\left[\Phi^{\alpha}\left(\frac{x_{i}-\mu}{\sigma}\right)\right]\right\} \\
& -\sum_{i=1}^{n} \log \left\{\frac{-\left[\Phi^{\alpha}\left(\frac{x_{i}-\mu}{\sigma}\right)\right]}{1-\left[\Phi^{\alpha}\left(\frac{x_{i}-\mu}{\sigma}\right)\right]}\right\}-(\beta-1) \sum_{i=1}^{n} \log \left\{1-\exp \left\{\frac{-\Phi^{\alpha}\left[\frac{x_{i}-\mu}{\sigma}\right]}{1-\Phi^{\alpha}\left[\frac{x_{i}-\mu}{\sigma}\right]}\right\}\right\} .
\end{aligned}
$$

Next, to demonstrate the feasibility of the suggested estimation strategy, a small simulation study was undertaken. The simulation study was carried out with $(\alpha, \beta, \mu, \sigma)=(1.7,2.5,3.2,0.8)$, respectively and the process was repeated 30,000 times. Three diff erent sample sizes $n=50,100$ and 200 were considered. The bias (actualestimate) and the standard deviation of the parameter estimates for the maximum likelihood estimates were determined from this simulation study and are presented in Table 5.

Table 5. Bias and standard deviation of the parameter estimates.

\begin{tabular}{|c|c|c|c|}
\hline Parameter & Sample size $(n=50)$ & Sample size $(n=100)$ & Sample size $(n=200)$ \\
\hline$\alpha$ & $0.1348(0.3548)$ & $0.0769(0.2271)$ & $0.0651(0.1718)$ \\
\hline$\beta$ & $0.2805(0.5673)$ & $0.1743(0.3496)$ & $0.0927(0.1154)$ \\
\hline$\mu$ & $0.0163(0.03534)$ & $-0.0134(0.02723)$ & $0.0135(0.01263)$ \\
\hline$\sigma$ & $0.0962(0.0452)$ & $0.08782(0.0389)$ & $0.0545(0.0217)$ \\
\hline
\end{tabular}

\subsection{Simulation for GOGE -Kumaraswamy distribution}

In this case the log-likelihood function is (from (6))

$$
\begin{aligned}
\ell= & n(\log \alpha+\log \beta+\log a+\log b)-(a-1) \sum_{i=1}^{n} x_{i}+(b-1) \sum_{i=1}^{n} \log \left(1-x_{i}^{a}\right) \\
& +(\alpha-1) \sum_{i=1}^{n} \log 1-\left(1-x_{i}^{a}\right)^{b}-2 \sum_{i=1}^{n} \log \left(1-\left[1-\left(1-x_{i}^{a}\right)^{b}\right]^{\alpha}\right) \\
& -\sum_{i=1}^{n} \log \left\{-\frac{\left[1-\left(1-x_{i}^{a}\right)^{b}\right]^{\alpha}}{1-\left[1-\left(1-x_{i}^{a}\right)^{b}\right]^{\alpha}}\right\}+(\beta-1) \sum_{i=1}^{n} \log \left\{1-e^{\left[-\frac{\left[1-\left(1-x_{i}^{a}\right)^{b}\right]^{\alpha}}{1-\left[1-\left(1-x_{i}^{a}\right)^{b}\right]^{\alpha}}\right]}\right\} .
\end{aligned}
$$


Next, to illustrate the feasibility of the suggested estimation strategy, a small simulation study was undertaken. The simulation study was carried out for one representative set of parameters $(\alpha, \beta, a, b)=(1.7,2.5,1.8,1.6)$ and the process was repeated 30,000 times. Three different sample sizes $n=50,100$ and 200 were considered. The bias (actual-estimate) and the standard deviation of the parameter estimates for the maximum likelihood estimates were determined from this simulation study and are presented in Table 6.

Table 6.Bias and standard deviation of the parameter estimates.

\begin{tabular}{|c|c|c|c|}
\hline Parameter & Sample size $(n=50)$ & Sample size $(n=100)$ & Sample size $(n=200)$ \\
\hline$\alpha$ & $0.1108(0.5382)$ & $0.0614(0.2345)$ & $0.0437(0.1139)$ \\
\hline$\beta$ & $0.1678(0.4628)$ & $-0.1321(0.1894)$ & $0.0672(0.0933)$ \\
\hline$a$ & $0.0268(0.4321)$ & $0.1483(0.2467)$ & $0.0946(0.1264)$ \\
\hline$b$ & $0.0825(0.0667)$ & $0.0779(0.0627)$ & $0.0621(0.0358)$ \\
\hline
\end{tabular}

\section{Conclusions}

A new class of distributions called the GOGE-G with two extra positive parameters has been introduced and studied, We provide some mathematical properties of the new family including entropies, probability weighted moments, residual life and reversed residual life functions, ordinary and incomplete moments, generating function and order statistics. Characterizations based on hazard function and as well as based on conditional expectation are presented. The maximum likelihood method is used for estimating the model parameters. A simple type Copula is constructed and a useful stochastic property is introduced. We assess the performance of the maximum likelihood estimators in terms of biases and mean squared errors by means of a simulation study. Finally, the usefulness of the family is illustrated using two real data sets.

\section{References}

[1] Aarset, M. V. (1987). How to identify bathtub hazard rate. IEEE Transactions on Reliability, 36, 106-108.

[2] Afify A. Z., Alizadeh, M., Yousof, H. M., Aryal, G. and Ahmad, M. (2016a). The transmuted geometric-G family of distributions: theory and applications. Pakistan Journal of Statistics, 32, 139-160.

[3] Afify, A. Z., Cordeiro, G. M., Nadarajah, S., Yousof, H. M., Ozel, G., Nofal, Z. M. and Emrah Altun. (2016b). The complementary geometric transmuted$\mathrm{G}$ family of distributions: model, properties and applications. Hacettepe Journal of Mathematics and Statistics, forthcoming.

[4] Afify A. Z., Cordeiro, G. M., Yousof, H. M., Alzaatreh, A. and Nofal, Z. M. (2016c). The Kumaraswamy transmuted-G family of distributions: properties and applications. Journal of Data Science, 14, 245-270. 
[5] Afify, A. Z., Yousof, H. M. and Nadarajah, S. (2017). The beta transmuted-H family of distributions: properties and applications. Stasistics and its Inference, 10, 505-520.

[6] Alizadeh, M., Cordeiro, G.M., Nascimento, A. D. C., Lima M. D. S. and Ortega, E. M. M. (2016a). Odd-Burr generalized family of distributions with some applications. Journal of Statistical Computation and Simulation, forthcoming.

[7] Alizadeh, M., Rasekhi, M., Yousof, H.M. and Hamedani, G.G. (2016b). The transmuted Weibull G family of distributions. Hacettepe Journal of Mathematics and Statistics, forthcoming.

[8] Alizadeh, M., Yousof, H. M., Afify, A. Z., Cordeiro, G.M. and Mansoor, M. (2016b). The complementary generalized transmuted Poisson-G family of distributions. Austrian Journal of Statistics, forthcoming.

[9] Alzaatreh, A., Lee, C. and Famoye, F. (2013). A new method for generating families of continuous distributions. Metron, 71, 63-79.

[10] Aryal, G. R. and Yousof, H. M. (2017). The exponentiated generalized-G Poisson family of distributions. Economic Quality Control, forthcoming.

[11] Barreto-Souza, W. et al. (2011). The Weibull-geometric distribution. Journal of Statistical Computation and Simulation, 60, 35-42.

[12] Bekker, A., Roux, J. and Mostert, P. (2000). A generalization of the compound Rayleigh distribution: using a Bayesian methods on cancer survival times. Commun. Stat. Theory Methods, 29, 1419-1433.

[13] Birnbaum, Z. W. and Saunders, S. C. (1969a). A new family of life distributions. Journal of Applied Probability, 6, 319-327.

[14] Birnbaum, Z. W. and Saunders, S. C. (1969b). Estimation for a family of life distributions with applications to fatigue. Journal of Applied Probability, 6, 328-377.

[15] Burnham, K. P. and Anderson, D. R. (2002), Model Selection and Multimodel Inference: A Practical Information-Theoretic Approach (2nd ed.) Springer-Verlag.

[16] Cooray, K. and Ananda, M. M. A. (2008). A Generalization of the HalfNormal Distribution with Applications to Lifetime Data. Communications in Statistics - Theory and Methods, 37, 1323-1337.

[17] Cordeiro, G. M. and Bager, R. S. B. (2012a). The beta power distribution. Brazilian Journal of Probability and Statistics, 26, 88-112. 
[18] Cordeiro, G. M., Hashimoto, E. M. and Ortega, E. M. M. (2014a). McDonald Weibull model. Statistics: A Journal of Theoretical and Applied Statistics, 48, 256-278.

[19] Cordeiro, G. M., Afify, A. Z., Yousof, H. M., Pescim, R. R. and Aryal, G. R. (2017a). The exponentiated Weibull-H family of distributions: Theory and Applications. Mediterranean Journal of Mathematics, forthcoming.

[20] Cordeiro, G. M., Ortega, E. M. M. and Nadarajah, S. (2010). The Kumaraswamy Weibull distribution with application to failure data. Journal of the Franklin Institute, 349, 11174-1197.

[21] Cordeiro, G. M., Afify, A. Z., Yousof, H. M., Pescim, R. R. and Aryal, G. R. (2017a). The exponentiated Weibull-H family of distributions: Theory and Applications. Mediterranean Journal of Mathematics, forthcoming.

[22] Cordeiro, G. M., Ortega, E. M. M. and Nadarajah, S. (2010). The Kumaraswamy Weibull distribution with application to failure data. Journal of the Franklin Institute, 349, 11174-1197.

[23] Cordeiro, G. M., Ortega, E. M. and da Cunha, D. C. C. (2013). The exponentiated generalized class of distributions. Journal of Data Science, 11, 127.

[24] Cordeiro, G. M., Pescim R. R and Ortega E. M. M. (2012b) The Kumaraswamy generalized half-normal distribution for skewed positive data. Journal of Data Science, 10. 195-224

[25] Cordeiro, G. M., Yousof, H. M., Ramires, T. G. and Ortega, E. M. M. (2017b). The Burr XII system of densities: properties, regression model and applications. Journal of Statistical Computation and Simulation, forthcoming.

[26] de Brito, E., Cordeiro, G. M., Yousof, H. M., Alizadeh, M. and Silva , G. O. (2017). Topp-Leone odd log-logistic family of distributions, Journal of Statistical Computation and Simulation, forthcoming.

[27] Famoye, F., Lee, C. and Olumolade, O. (2005). The beta-Weibull distribution. Journal of Statistical Theory and Applications, 4, 121-136

[28] Gl"anzel, W. (1987). A characterization theorem based on truncated moments and its application to some distribution families, Mathematical Statistics and Probability Theory (Bad Tatzmannsdorf, (1986), Vol. B, Reidel, Dor-drecht, 75-84.

[29] Gl"anzel, W. (1990). Some consequences of a characterization theorem based on truncated moments, Statistics: A Journal of Theoretical and Applied Statistics, 21, 613-618. 
[30] Gupta, R. C., Gupta, P. L. and Gupta, R. D. (1998). Modeling failure time data by Lehmann alternatives. Communications in Statistics - Theory and Methods, 27, 887-904.

[31] Hamedani G. G. Yousof, H. M., Rasekhi, M., Alizadeh, M., Najibi, S. M. (2017). Type I general exponential class of distributions. International Journal of Applied \& Experimental Mathematics, forthcoming.

[32] Louzada, F., Marchi, V. and Roman, M. (2012). The exponentiated exponential geometric distribution: a distribution with decreasing, increasing and unimodal failure rate. Statistics: A Journal of Theoretical and Applied Statistics, 48, 167-181.

[33] Marshall, A. W. and Olkin, I. (1997). A new methods for adding a parameter to a family of distributions with application to the Exponential and Weibull families. Biometrika, 84, 641-652.

[34] Mudholkar, G. S. and Srivastava, D. K. (1993). Exponentiated Weibull family for analysing bathtub failure rate data. IEEE Transactions on Reliability, 42, 299-302.

[35] Nadarajah, S. (2005). The exponentiated Gumbel distribution with climate application. Environmetrics, 17, 13-23.

[36] Nadarajah, S. and Kotz, S. (2006). The beta exponential distribution. Reliability Engineering and System Safety, 91, 689-697.

[37] Nelsen, R. B. (1999). An Introduction to Copulas. Springer-Verlag, New York.

[38] Nofal, Z. M., Afify, A. Z., Yousof, H. M. and Cordeiro, G. M. (2017). The gener-alized transmuted-G family of distributions. Communications in Statistics - Theory and Methods, 46, 4119-4136.

[39] Pougaza, D. B. and Djafari, M. A. (2011). Maximum entropies copulas. Proceedings of the 30th international workshop on Bayesian inference and maximum Entropy methods in Science and Engineering, 329-336.

[40] Ristic, M. M., Balakrishnan, N. (2012). The gamma-exponentiated exponential distribution. Journal of Statistical Computation and Simulation, 82, 1191-1206.

[41] Yousof, H. M., Afify, A. Z., Alizadeh, M., Butt, N. S., Hamedani, G. G. and Ali, M. M. (2015). The transmuted exponentiated generalized-G family of distributions. Pakistan Journal of Statistics and Operation Research, 11, 441464.

[42] Yousof, H. M., Afify, A. Z., Alizadeh, M., Nadarajah, S., Aryal, G. R. and Hamedani, G. G. (2017a). The Marshall-Olkin generalized-G family of 
[43] distributions with Applications. Communications in Statistics-Theory and Methods, forthcoming.

[44] Yousof, H. M., Afify, A. Z., Hamedani, G. G. and Aryal, G. (2016). the Burr $\mathrm{X}$ generator of distributions for lifetime data. Journal of Statistical Theory and Applications, forthcoming.

[45] Yousof, H. M., Rasekhi, M., Afify, A. Z., Alizadeh, M., Ghosh, I. and Hamedani G. G. (2017b). The beta Weibull-G family of distributions: theory, charac-terizations and applications, Pakistan Journal of Statistics, 33, 95-116.

${ }^{1}$ Morad Alizadeh

Department of Statistics

Faculty of Sciences, Persian Gulf University, Iran

${ }^{2}$ Indranil Ghosh

Department of Mathematics and Statistics

University of North Carolina, USA

${ }^{3}$ Haitham M. Yousof

Department of Statistics Mathematics and Insurance

Benha University, Egypt

${ }^{4}$ Mahdi Rasekhi

Department of Statistics

Malayer University, Iran

${ }^{5}$ G.G.Hamedani

DepartmentofMathematics, Statistics and Computer Science, Marquette University, USA 
